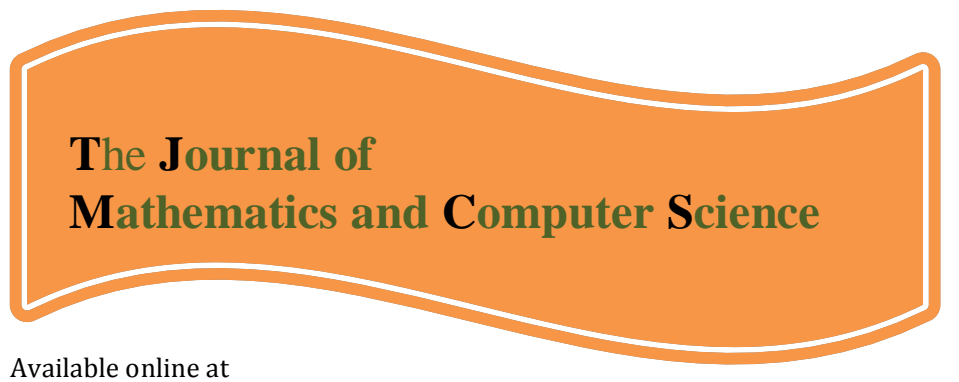

http://www.TJMCS.com

The Journal of Mathematics and Computer Science Vol .2 No.1 (2011) 184-194

\title{
Fuzzy Cost Analysis in a Fuzzy Transportation System: a Study of the Supply Chain Management in a General Contractor Company
}

\author{
Hossein Abdollahnejad Barough ${ }^{1,2 *}$ \\ hosseinab@kayson-ir.com
}

Received: September 2010, Revised: November 2010

Online Publication: January 2011

\begin{abstract}
Transportation models play an important role in logistics and supply chain management for reducing cost and improving services. In this paper, the author presented a fuzzy transportation problem, in which the cost coefficients and supply and demand quantities are fuzzy numbers. The problem is solved in two stages. First, calculating the maximum satisfactory level and achieving balances between fuzzy supplies and demands. Second, the problem is solved by considering the unit of transportation costs and optimal solutions which are connected with fuzzy quantities' satisfactory level are founded. The author used two different satisfactory levels for the problem: The transportation costs breaking points $\left(\gamma_{p}\right)$ and the values that have violated positive condition of optimal solutions in the intervals of $\left[\gamma_{\mathrm{p}-1}, \gamma_{\mathrm{p}}\right]$. A new method is proposed in this paper to find optimal solutions. The proposed method is then illustrated through a numerical example.
\end{abstract}

Keywords: Fuzzy Transportation Problem, Supply Chain Management, Fuzzy Cost Analysis, Linear Programming.

\section{Introduction}

The transportation problem is a linear programming problem stemmed from a network structure consisting of a finite number of nodes and arcs attached to them that transports a homogenous product from $\mathrm{M}$ sources to $\mathrm{N}$ different destinations to minimize total transportation

$1,{ }^{*}$ Corresponding author: Department of Industrial Engineering, Payam-e-Noor University.

$2,{ }^{*}$ System Analyst, Planning and System Department, Kayson Co. 
cost. There are numerous solution methods for transportation problem when prices and quantities are given as crisp numbers [8], [10]. Several variations of transportation methods have been using with the table methods such as the northwest corner method, the shortcut method and Russell's approximation method [8], [10]. Some others [2], [3], [17] have been using special techniques for linear programming (LP) problem because the classic single objective transportation problem is a special case of the linear programming problem. However, recently fuzzy programming approach started to use the optimal solutions of multi objective or single objective transportation problem [4], [9], [11], [16], [17]. For instance, Wahed [16] presented a fuzzy programming approach to determine the compromise solution of multi objective transportation problem (MOTP). Kikuchi [9] proposed a simple adjustment method that finds the most appropriate set of crisp numbers. Wahed et al. [17] presented an interactive fuzzy goal programming approach to determine the preferred compromise solution for MOTP. In reality, it is not possible to determine both quantities and transportation unit prices, but the fuzzy numbers gives best approximation of them. A model solving the transportation problem is given in [11] when quantities are fuzzy and prices are crisp. The model uses the table method for solution. Again, in [9] is given a method determining quantities that is satisfied the higher satisfactory level while quantities is only fuzzy. This method uses LP model in solution. OhEigeartaigh [10], [14] considered the case where the membership functions of the fuzzy demands are triangular forms for transportation problems and solved it using table method. Chanas and Kulej [3], [10] provided an approach to solve a fuzzy linear programming problem with triangular membership functions of fuzzy resources. Geetha and Nair [6] formulated a stochastic version of the time minimizing transportation problem and developed an algorithm based on parametric programming to solve it when transportation time is considered to be independent, positive normal random variables. Chanas et al. [4] are analyzed the transportation problem with fuzzy supply values of deliverers and with fuzzy demand values of the receivers. Liu and Kao [13] developed a procedure to derive the fuzzy objective value of the fuzzy transportation problem, in that the cost coefficients and the supply and demand quantities are fuzzy numbers basing on extension principle. Ahlatcioglu, Sivri and Güzel [1] proposed a solution algorithm finding all fuzzy optimal solution of the transportation problem that the cost coefficients and the supply and demand quantities are fuzzy numbers. Chanas and Kuchta [5] proposed an algorithm that solves the transportation problem with fuzzy supply and demand values and integrality condition imposed on the solution. Ammar and Youness [2] investigated the efficient solutions and stability of fuzzy multi-objective transportation problem and proposed an algorithm for the determination of the stability set. Gen etal. [7] describes an implementation of genetic algorithm to solve Bicriteria Solid Transportation Problem (BSTP). Li et al. [12] proposed neural network approach for multi-criteria solid transportation problem.

In this paper, the author presented a fuzzy transportation problem in which the cost coefficients and supply and demand quantities are triangular fuzzy numbers. The problem is solved in two stages. First, calculating the maximum satisfactory level which achieves balances between fuzzy supplies and demands. In this stage the traditional transportation problem is developed according to the maximum satisfactory level. Second, the problem is solved by considering the unit of transportation costs and optimal solutions which connected with fuzzy quantities' satisfactory level are founded with the help of breaking points of the unit of transportation costs that varies from zero to the maximum satisfactory level. Two different satisfactory levels were used for the problem: the braking points $\left(\gamma_{p}\right)$ of transportation costs $\left(\tilde{C}_{i j}=\tilde{C}_{i j}(\gamma)\right)$ and the $\left(\alpha_{s}\right)$, the value that violate positive condition of optimal solutions in the intervals of $\left[\alpha_{s-1}, \alpha_{s}\right]$ on $\left[\gamma_{p-1}, \gamma_{p}\right]$.The organization of this paper is as follows. Section two reviews some basic definitions and assumptions of the area of fuzzy theory. Section three studies the problem formulation. Section four considers the definition 
of cost prices' braking points, crisp cost prices are founded in this section and it explains the solution method. One modified real numerical example of proposed method is presented in section five and finally section six gives some concluding remarks on the proposed method.

\section{Definitions and Assumptions}

In this section, some basic notions of the area of fuzzy theory that have been defined by Kaufmaan and Gupta (1985) and Zimmermann (1996) are introduced.

Definition1: Let $\mathrm{R}$ be the space of real numbers. A Fuzzy set $\tilde{A}_{i}$ is a set of ordered $\operatorname{pairs}\left\{\left(x, \mu_{\widetilde{A}_{i}}(x) \mid x \in R\right)\right\}$, where $\mu_{\widetilde{A}_{i}}(\mathrm{x}): \rightarrow[0,1]$ and is upper semi continuous. Function $\mu_{\widetilde{A}_{i}}(x)$ is called membership function of the fuzzy set.

Definition 2: A convex fuzzy set, $\tilde{\mathrm{A}}_{\mathrm{i}}$, is a fuzzy set in which:

$\forall x, y \in R, \forall \lambda \in[0,1]$,

$\mu_{\widetilde{A}_{i}}(\lambda x+(1-\lambda) y) \geq \min \left[\mu_{\widetilde{A}_{i}}(x), \mu_{\widetilde{A}_{i}}(y)\right]$

Definition3: A fuzzy set $\tilde{A}$ is called positive if its membership function is such that $\mu_{\tilde{A}_{i}}(x)=0, \forall x \leq$ 0 .

Definition4: Triangular Fuzzy Number (TFN) is a convex fuzzy set which is defined as

$\tilde{A}=\left(x, \mu_{\tilde{A}_{i}}(x)\right)$ Where:

$\mu_{\widetilde{\mathrm{A}}_{\mathrm{i}}}(x)=\left\{\begin{array}{c}0, \quad x \leq a_{i}^{1} \\ \frac{\left(x-a_{i}^{1}\right)}{\left(a_{i}^{2}-a_{i}^{1}\right)}, a_{i}^{1}<x \leq a_{i}^{2} \\ \frac{\left(x-a_{i}^{3}\right)}{\left(a_{i}^{2}-a_{i}^{3}\right)}, a_{i}^{2}<x \leq a_{i}^{3} \\ 0, \quad x>a_{i}^{3}\end{array}\right.$

For convenience, TFN represented by three real parameters $a_{1}, a_{2}, a_{3}$ which are $\left(a_{i}^{1} \leq a_{i}^{2} \leq a_{i}^{3}\right)$ Will be denoted by tetraploid $a_{i}^{1}, a_{i}^{2}, a_{i}^{3}$ (Fig.1).

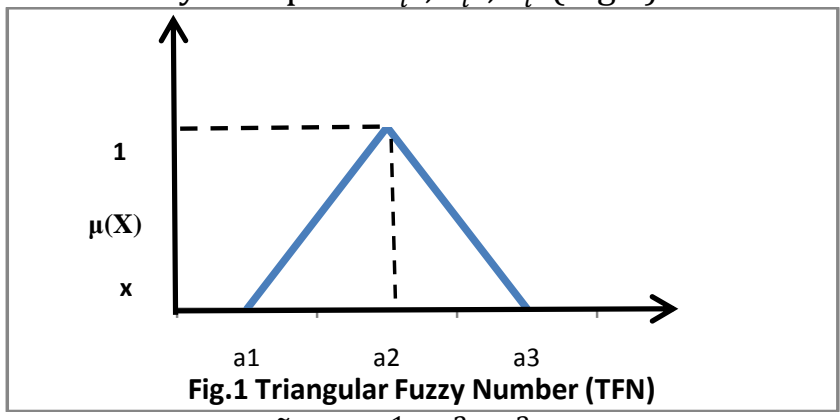

Definition5: A triangular fuzzy number $\tilde{A}_{i}=\left(a_{i}^{1}, a_{i}^{2}, a_{i}^{3}\right)$ is called positive TFN if: $0 \leq a_{i}^{1} \leq a_{i}^{2} \leq a_{i}^{3}$

\section{Problem Formulation}

Transportation problem that examined here can be described as follows. Consider the traditional transformation problem with homogenous product from $\mathrm{M}$ sources to $\mathrm{N}$ different destinations and a target of minimizing total transportation costs but with crisp numbers.

\subsection{Notations}

The following notations are applied to describe the transportation problem:

Indices and parameters: 


$\begin{array}{ll}\mathrm{M} & \text { Number of sources } \\ \mathrm{N} & \text { Number of destinations } \\ \tilde{A}_{i} & \text { Fuzzy quantity of } \mathrm{i}^{\text {th }} \text { source } \\ \tilde{B}_{j} & \text { Fuzzy quantity of } \mathrm{j}^{\text {th }} \text { destination } \\ \tilde{C}_{i j} & \text { Fuzzy unit transportation cost from } \mathrm{i}^{\text {th }} \text { source to } \mathrm{j}^{\text {th }} \text { destination } \\ \alpha & \text { The possibility level of supply and demand } \\ \gamma & \text { The possibility level of the unit of transportation cost } \\ \text { Decision variables: } & \text { Fuzzy quantities which is transported from } \mathrm{i}^{\text {th }} \text { source to } \mathrm{j}^{\text {th }} \\ \tilde{X}_{i j} & \text { destination }\end{array}$

\subsection{Fuzzy Transportation Linear Programming (FTLP) Model}

\subsubsection{The Objective Programming Function}

The objective programming function consists of total transportation costs. The related cost coefficients in the objective function are frequently imprecise in nature because some information is incomplete or unobtainable. Accordingly the objective function of the proposed model is as follows:

Minimize $\tilde{Z}=\sum_{i=1}^{m} \sum_{j=1}^{n} \tilde{C}_{i j} \tilde{X}_{i j}$

\subsubsection{Constraints}

The constraints are as follows:

$\sum_{j=1}^{n} \tilde{X}_{i j} \cong\left(a_{i}^{1}, a_{i}^{2}, a_{i}^{3}\right), \quad(i=1,2, \ldots ., m)$

$\sum_{i=1}^{m} x_{i j} \cong\left(b_{j}^{1}, b_{j}^{2}, b_{j}^{3}\right), \quad(j=1,2, \ldots . ., n)$

$\tilde{x}_{i j} \geq 0$ and are integers

$\sum_{i} \tilde{A}_{i j}=\sum_{j} \tilde{B}_{i j}$

\section{The Solution Procedure}

$\tilde{A}_{i}$ and $\tilde{B}_{j}$ are fuzzy quantities and shown as $\tilde{A}_{i}=\left(a_{i}^{1}, a_{i}^{2}, a_{i}^{3}\right)$ and $\tilde{B}_{j}=\left(b_{j}^{1}, b_{j}^{2}, b_{j}^{3}\right)$ fuzzy triangle numbers. The unit transportation cost is given as fuzzy number $\tilde{C}_{i j}=\left(-\infty, \tilde{C}_{i j}^{2}, \tilde{C}_{i j}^{3}\right)$. Let $\mu_{\tilde{A}_{i}}, \mu_{\tilde{B}_{j}}$ and $\mu_{\tilde{C}_{i j}}$ denote the membership function as below

$\tilde{A}_{i}=\left\{\left(a_{i}, \mu_{\tilde{A}_{i}}\right) \mid a_{i} \in A\left(\tilde{A}_{i}\right)\right\}$

$\tilde{B}_{j}=\left\{\left(b_{j}, \mu_{\tilde{B}_{j}}\right) \mid b_{j} \in B\left(\tilde{B}_{j}\right)\right\}$

$\tilde{C}_{i j}=\left\{\left(c_{i j}, \mu_{\tilde{C}_{i j}}\right) \mid c_{i j} \in C\left(\tilde{C}_{i j}\right)\right\}$

Where $A\left(\tilde{A}_{i}\right), B\left(\tilde{B}_{j}\right), C\left(\tilde{C}_{i j}\right)$ are the support of $\tilde{A}_{i}, \tilde{B}_{j}, \tilde{C}_{i j}$. Denote the $\alpha$ - cut of $\tilde{A}_{i}, \tilde{B}_{j}$ and the $\gamma$ - cut of $\tilde{C}_{i j}$ as: 


$$
\begin{aligned}
& \left(\tilde{A}_{i}\right)_{\alpha}=\left[\left(\tilde{A}_{i}\right)_{\alpha}^{L},\left(\tilde{A}_{i}\right)_{\alpha}^{U}\right] \\
& =\left[\min _{\tilde{A}_{i}}\left\{a_{i} \in A\left(\tilde{A}_{i}\right) \mid \mu_{\tilde{A}_{i}} \geq \alpha\right\}, \tilde{A}_{i}\left\{a_{i} \in A\left(\tilde{A}_{i}\right) \mid \mu_{\tilde{A}_{i}} \geq \alpha\right\}\right] \\
& =\left[\tilde{A}_{i}^{1}+\alpha\left(\tilde{A}_{i}^{2}-\tilde{A}_{i}^{1}\right), \tilde{A}_{i}^{3}-\alpha\left(\tilde{A}_{i}^{3}-\tilde{A}_{i}^{2}\right)\right] \\
& \left(\tilde{B}_{j}\right)_{\alpha}=\left[\left(\tilde{B}_{j}\right)_{\alpha}^{L},\left(\tilde{B}_{j}\right)_{\alpha}^{U}\right] \\
& =\left[\min _{\tilde{B}_{j}}\left\{b_{j} \in B\left(\tilde{B}_{j}\right) \mid \mu_{\tilde{B}_{j}} \geq \alpha\right\}, \tilde{B}_{j}\left\{b_{j} \in B\left(\tilde{B}_{j}\right) \mid \mu_{\tilde{B}_{j}} \geq \alpha\right\}\right] \\
& =\left[\tilde{B}_{j}^{1}+\alpha\left(\tilde{B}_{j}^{2}-\tilde{B}_{j}^{1}\right), \tilde{B}_{j}^{3}-\alpha\left(\tilde{B}_{j}^{3}-\tilde{B}_{j}^{2}\right)\right] \\
& \left(\tilde{C}_{i j}\right)_{\gamma}=\left[-\infty,\left(\tilde{C}_{i j}\right)_{\gamma}^{U}\right] \\
& =\left[-\infty, \tilde{C}_{i j}\left\{c_{i j} \in C\left(\tilde{C}_{i j}\right) \mid \mu_{\tilde{C}_{i j}} \geq \gamma\right\}\right] \\
& =\left[-\infty, \tilde{C}_{i j}^{3}-\gamma\left(\tilde{C}_{i j}^{3}-\tilde{C}_{i j}^{2}\right)\right]
\end{aligned}
$$

These intervals indicate where supply and demand lie at possibility level of $\alpha$ and the unit of transportation cost lie at possibility level of $\gamma$.

In the first step, based on the extension principle [5],the membership function of supply and demand can be defined as:

Maximiz $\alpha$

Subject to

$\tilde{A}_{i}^{1}+\alpha\left(\tilde{A}_{i}^{2}-\tilde{A}_{i}^{1}\right) \leq \sum_{j=1}^{n} \tilde{X}_{i j} \leq \tilde{A}_{i}^{3}-\alpha\left(\tilde{A}_{i}^{3}-\tilde{A}_{i}^{2}\right)$

$\tilde{B}_{j}^{1}+\alpha\left(\tilde{B}_{j}^{2}-\tilde{B}_{j}^{1}\right) \leq \sum_{i=1}^{m} \tilde{X}_{i j} \leq \tilde{B}_{j}^{3}-\alpha\left(\tilde{B}_{j}^{3}-\tilde{B}_{j}^{2}\right)$

$\tilde{X}_{i j} \geq 0$ for $i=(1,2, \ldots) \& j=(1,2, \ldots .$.

From optimal solution of problem (2.5) we obtain $(\bar{\alpha}=$ Maximize $\alpha)$.

For supplies:

$\sum_{j=1}^{n} \tilde{X}_{i j} \leq \tilde{A}_{i}^{2}$

then the related constraints are rearranged as:

$\sum_{j=1}^{n} \tilde{X}_{i j}=\tilde{A}_{i}^{1}+\alpha\left(\tilde{A}_{i}^{2}-\tilde{A}_{i}^{1}\right) \&(i=1,2, \ldots ., m)$

Otherwise the related constraints are rearranged as:

$\sum_{j=1}^{n} \tilde{X}_{i j}=\tilde{A}_{i}^{3}-\alpha\left(\tilde{A}_{i}^{3}-\tilde{A}_{i}^{2}\right) \&(i=1,2, \ldots ., m)$

For demands :

$\sum_{j=1}^{n} \tilde{X}_{i j} \leq \tilde{B}_{j}^{2}$

then the related constraints are rearranged as:

$\sum_{j=1}^{n} \tilde{X}_{i j}=\tilde{B}_{j}^{1}+\alpha\left(\tilde{B}_{j}^{2}-\tilde{B}_{j}^{1}\right)$

Otherwise the related constraints are rearranged as: 
$\sum_{j=1}^{n} \tilde{X}_{i j}=\tilde{B}_{j}^{3}-\alpha\left(\tilde{B}_{j}^{3}-\tilde{B}_{j}^{2}\right) \&(j=1,2, \ldots ., n)$

In the first step, supplies and demands have rearranged according to the supply and demand possibility level $(\alpha)$. In second step, from twice intersection of the unit of transportation costs in each cells relating to the unit of transportation cost possibility level $(\gamma)$,. we obtain breaking points of costs.

\subsection{The Membership Function}

Membership functions of each fuzzy numbers $\tilde{A}_{i}, \tilde{B}_{j}, \tilde{C}_{i j}$ can be written as:

$\mu_{\tilde{A}_{i}}=\left\{\begin{array}{cl}0, & \tilde{A}_{i} \leq \tilde{A}_{i}^{1} \\ \frac{\left(\tilde{A}_{i}-\tilde{A}_{i}^{1}\right)}{\left(\tilde{A}_{i}^{2}-\tilde{A}_{i}^{1}\right)}, & \tilde{A}_{i}^{1} \leq \tilde{A}_{i} \leq \tilde{A}_{i}^{2} \\ \frac{\left(\tilde{A}_{i}-\tilde{A}_{i}^{3}\right)}{\left(\tilde{A}_{i}^{2}-\tilde{A}_{i}^{3}\right)}, & \tilde{A}_{i}^{2} \leq \tilde{A}_{i} \leq \tilde{A}_{i}^{3} \\ 0, & \tilde{A}_{i} \geq \tilde{A}_{i}^{3}\end{array}\right.$

$\mu_{\tilde{B}_{j}}=\left\{\begin{array}{cl}0, & \tilde{B}_{j} \leq \tilde{B}_{j}^{1} \\ \frac{\left(\tilde{B}_{j}-\tilde{B}_{j}^{1}\right)}{\left(\tilde{B}_{j}^{2}-\tilde{B}_{j}^{1}\right)}, & \tilde{B}_{j}^{1} \leq \tilde{B}_{j} \leq \tilde{B}_{j}^{2} \\ \frac{\left(\tilde{B}_{j}-\tilde{B}_{j}^{3}\right)}{\left(\tilde{B}_{j}^{2}-\tilde{B}_{j}^{3}\right)}, & \tilde{B}_{j}^{2} \leq \tilde{B}_{j} \leq \tilde{B}_{j}^{3} \\ 0, & \tilde{B}_{j} \geq \tilde{B}_{j}^{3}\end{array}\right.$

$\mu_{\tilde{C}_{i j}}=\left\{\begin{array}{c}1, \tilde{C}_{i j} \leq \tilde{C}_{i j}^{2} \\ \frac{\tilde{C}_{i j}-\tilde{C}_{i j}^{3}}{\tilde{C}_{i j}^{2}-\tilde{C}_{i j}^{3}}, \tilde{C}_{i j}^{2} \leq \tilde{C}_{i j} \leq \tilde{C}_{i j}^{3} \\ 0, \tilde{C}_{i j} \geq \tilde{C}_{i j}^{3}\end{array}\right.$

There is one $\tilde{A}_{i}=\mu_{i}^{-1}(\alpha)$ and $\tilde{B}_{j}=\mu_{j}^{-1}(\alpha)$ each of them exists in $\alpha \in[0, \bar{\alpha}]$, because the membership functions are monotone for $0<\alpha<1$, if these numbers are explained with $\alpha$-parameter, then we obtain:

$\tilde{A}_{i}^{-1}=\mu_{i}^{-1}(\alpha)=\tilde{A}_{i}^{1}+\left(\tilde{A}_{i}^{2}-\tilde{A}_{i}^{1}\right) \alpha$

And

$\tilde{A}_{i}^{-1}=\mu_{i}^{-1}(\alpha)=\tilde{A}_{i}^{3}+\left(\tilde{A}_{i}^{3}-\tilde{A}_{i}^{2}\right) \alpha$

From $\mu_{\tilde{A}_{i}}=\alpha$ and

$\tilde{B}_{j}=\mu_{j}^{-1}(\alpha)=\tilde{B}_{j}^{1}+\left(\tilde{B}_{j}^{2}-\tilde{B}_{j}^{1}\right) \alpha$

And

$\tilde{B}_{j}=\mu_{j}^{-1}(\alpha)=\tilde{B}_{j}^{3}+\left(\tilde{B}_{j}^{3}-\tilde{B}_{j}^{3}\right) \alpha$

From $\mu_{\tilde{B}_{j}}=\alpha$. The membership functions of these numbers are shown in Fig.2. 


\subsection{Equality Constraints}

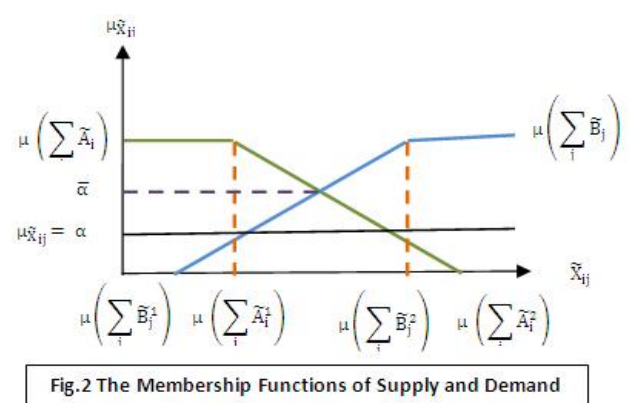

The equation (16) must satisfied at $(\bar{\alpha})$ level

$\bar{\alpha}=$ Maximize Minimize $\left[\mu\left(\sum_{i} \tilde{A}_{i}\right), \mu\left(\sum_{j} B_{j}\right)\right], \bar{\alpha} \in[0,1]$

For solving this problem, the problem can be balanced by adding an artificial source. As shown in Fig 3. $\alpha \in[0, \bar{\alpha}]$ could not show directly the satisfactory level in transportation problems and the problem is balanced at $(\bar{\alpha})$ level. The satisfactory level $(\alpha)$ can increase as much as $(\alpha=1)$ but in solution involving quantities out of the intervals the $(\alpha)$ value because $(\alpha<1)$.

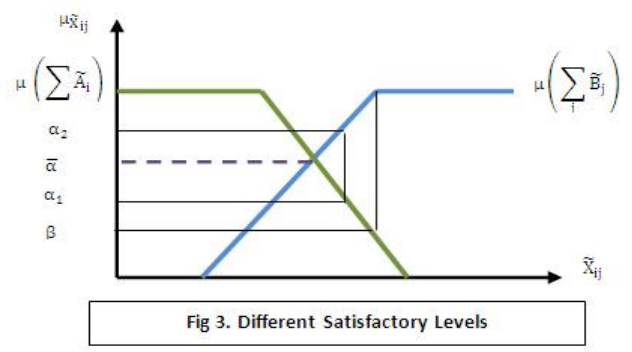

\subsection{Defining Braking Points of the Prices}

Because the membership function of $C_{i j}$ is decreasing in $\mu_{\tilde{C}_{i j}} \in(0,1)$ intervals we have:

$\mu_{\tilde{C}_{i j}}=\gamma$ So that we have $\tilde{C}_{i j}=\mu_{\gamma}^{-1}$

In this state, the transportation costs are defined in terms of satisfactory level $(\gamma)$, shown in Fig4.

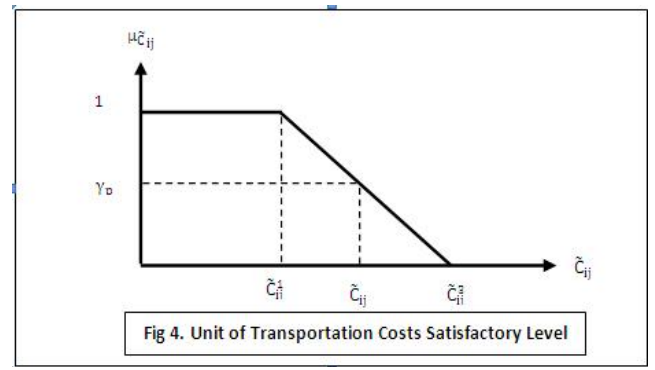

That is,

$\tilde{C}_{i j}=\tilde{C}_{i j}^{3}-\gamma\left(\tilde{C}_{i j}^{3}-\tilde{C}_{i j}^{2}\right)$

By means of the twice intersection of

$\tilde{C}_{i j}=\tilde{C}_{i j}^{3}-\gamma\left(\tilde{C}_{i j}^{3}-\tilde{C}_{i j}^{2}\right)$

lines, the $\mathrm{N}=$ m.n piece values of $\gamma \in[0,1]$ for each $\mathrm{k}$ are obtained and then these values change the order of $C_{i j}$.These values of $\gamma_{p}$ are called the breaking points [15]. The order of $\tilde{C}_{i j}$ 's in each subintervals that are formed between the iterative breaking points would not change. Therefore, the representative point can be selected in this interval. This selected point can be any point of the interval, but the optimal solution of transportation problem would not change. However, when the interval changes the consecutive order among the costs changes as well. Therefore, the optimal 
solution of transportation problem changes as a result of these changes. As a result, for each interval that is formed the iterative breaking points of $\gamma_{p}$ are searched the optimal solutions one by one. Thus, all optimal solutions are investigated.

Let this interval $\left[\gamma_{p-1}, \gamma_{p}\right]$ be subinterval by forming iterative breaking points. By selecting $\hat{\gamma}_{p} \in\left[\gamma_{p-1}, \gamma_{p}\right]$ we solve the transportation problem for crisp costs, by using $\tilde{C}_{i j}=\tilde{C}_{i j}^{3}-\gamma\left(\tilde{C}_{i j}^{3}-\tilde{C}_{i j}^{2}\right)$

As you know, for the transported quantity $\tilde{X}_{i j}$ from ith $^{\text {source to }} \mathrm{j}^{\text {th }}$ demand center the fuzzy transportation problem is stated as follows,

Minimize $\tilde{Z}=\sum_{i=1}^{m} \sum_{j=1}^{n} \tilde{C}_{i j} \tilde{X}_{i j}$

$\sum_{j=1}^{n} \tilde{X}_{i j}=\tilde{A}_{i}^{3}-\alpha\left(\tilde{A}_{i}^{3}-\tilde{A}_{i}^{2}\right)$

$\sum_{j=1}^{n} \tilde{X}_{i j}=\tilde{A}_{i}^{1}+\alpha\left(\tilde{A}_{i}^{2}-\tilde{A}_{i}^{1}\right)$

$\sum_{j=1}^{n} \tilde{X}_{i j}=\tilde{B}_{j}^{1}+\alpha\left(\tilde{B}_{j}^{2}-\tilde{B}_{j}^{1}\right)$

$\sum_{j=1}^{n} \tilde{X}_{i j}=\tilde{B}_{j}^{3}-\alpha\left(\tilde{B}_{j}^{3}-\tilde{B}_{j}^{2}\right)$

$\tilde{X}_{i j} \geq 0$ for $i=(1,2, \ldots) \& j=(1,2, \ldots \ldots)$

4.4. Defining of $(\alpha)$ Intervals

Depending on ? that changes in $[0,1]$ interval the quantities of source and demand centers and optimal solutions will change. Thus, optimal solutions $\tilde{X}_{i j}^{*}=\tilde{X}_{i j}^{*}(\alpha)$ are linear but while variations of ? change the transported quantities continuously, non-negative constraint will violate. The (国. which violates non-negative constraint of optimal solution is called breaking points of ?. After the value of (? ? . in this breaking point, at least one component of optimal solution is negative. Therefore, the feasible condition will violate. By using the dual problem the feasible condition is gained again. If the variation in the positive direction of (? ? again violates the feasible condition, same operations are continued. Therefore, different optimal solutions can be obtained as the number of intervals. When the costs and quantities are given fuzzy numbers, the numbers of optimal solutions are p. $r$, where $\mathrm{p}$ is the number of interval that is formed by $\gamma$ in $[0,1]$ and $r$ is the number of interval that is formed by $\alpha$ in $[0, \bar{\alpha}]$. But, some optimal solutions are same as the others.

\section{Numerical Example}

We consider the following modified fuzzy transportation problem that is a real case of a Supply Chain Management model in the Logistics Department of Kayson Co. Table.1 shows the information on the fuzzy quantity supplied and demanded as well as the fuzzy transportation costs per unit.The fuzzy quantities to be transported from the sources to the different destinations are represented by the decision variables $\left(\tilde{X}_{i 1}, \tilde{X}_{i 2}, \ldots, \tilde{X}_{1 j}, \tilde{X}_{2 j}, \ldots\right)$ in the cells.

Table.1: Transportation Supply, Demand and Cost Coefficients Fuzzy Values

\begin{tabular}{|c|c|c|c|}
\hline Demand & $(2,5,6)$ & $(14,15,17)$ & $(2,5,6)$ \\
\hline
\end{tabular}




\begin{tabular}{|c|c|c|c|}
\hline$(2,9,11)$ & $\tilde{\mathrm{C}}_{11}(-\infty, 3,5)$ & $\tilde{\mathrm{C}}_{12}(-\infty, 6,7)$ & $\tilde{\mathrm{C}}_{13}(-\infty, 6,11)$ \\
\hline$(3,8,12)$ & $\tilde{\mathrm{C}}_{21}(-\infty, 7,9)$ & $\tilde{\mathrm{C}}_{22}(-\infty, 9,15)$ & $\tilde{\mathrm{C}}_{23}(-\infty, 10,18)$ \\
\hline$(4,9,14)$ & $\tilde{\mathrm{C}}_{31}(-\infty, 9,13)$ & $\tilde{\mathrm{C}}_{32}(-\infty, 9,16)$ & $\tilde{\mathrm{C}}_{33}(-\infty, 9,10)$ \\
\hline
\end{tabular}

Now we consider transportation problem with fuzzy cost and fuzzy quantity.

$\operatorname{Max} \alpha$

Subject to

$\tilde{X}_{i 1}+\tilde{X}_{i 2}+\tilde{X}_{i 3} \geq 2+7 \alpha$

$\tilde{X}_{i 1}+\tilde{X}_{i 2}+\tilde{X}_{i 3} \leq 11-2 \alpha$

$\tilde{X}_{i 4}+\tilde{X}_{i 5}+\tilde{X}_{i 6} \geq 3+4 \alpha$

$\tilde{X}_{i 4}+\tilde{X}_{i 5}+\tilde{X}_{i 6} \leq 12-4 \alpha$

$\tilde{X}_{i 7}+\tilde{X}_{i 8}+\tilde{X}_{i 9} \geq 4+5 \alpha$

$\tilde{X}_{i 7}+\tilde{X}_{i 8}+\tilde{X}_{i 9} \leq 14-5 \alpha$

$\tilde{X}_{1 j}+\tilde{X}_{2 j}+\tilde{X}_{3 j} \geq 2+3 \alpha$

$\tilde{X}_{1 j}+\tilde{X}_{2 j}+\tilde{X}_{3 j} \leq 6-\alpha$

$\tilde{X}_{4 j}+\tilde{X}_{5 j}+\tilde{X}_{6 j} \geq 14+\alpha$

$\tilde{X}_{4 j}+\tilde{X}_{5 j}+\tilde{X}_{6 j} \leq 17-2 \alpha$

$\tilde{X}_{7 j}+\tilde{X}_{8 j}+\tilde{X}_{9 j} \geq 5+5 \alpha$

$\tilde{X}_{7 j}+\tilde{X}_{8 j}+\tilde{X}_{9 j} \leq 13-3 \alpha$

$\tilde{X}_{i j} \geq 0$ for $i=(1, \ldots, 9) \& j=(1, \ldots, 9)$

Solution of problem (22) is $\alpha=0.8$ and $\tilde{X}_{11}=3.4, \tilde{X}_{12}=6, \tilde{X}_{13}=0, \tilde{X}_{21}=0, \tilde{X}_{22}=8.8, \tilde{X}_{23}=$ $0, \tilde{X}_{31}=1, \tilde{X}_{32}=0, \tilde{X}_{33}=9 . \alpha_{1}=9.4, \alpha_{2}=8.8, \alpha_{3}=10$ and $b_{j}^{1}=4.4, b_{j}^{2}=14.8, b_{j}^{3}=9$

Where $9 \leq \alpha_{1} \leq 11,8 \leq \alpha_{2} \leq 12,9 \leq \alpha_{3} \leq 14$ and $2 \leq b_{j}^{1} \leq 5,14 \leq b_{j}^{2} \leq 15,5 \leq b_{j}^{3} \leq 10$.

From this solution we obtain the given fuzzy transportation problem can be rearranged as the fuzzy transportation problem in Table 2. where quantities in artificial demand center are

$\sum_{i=1}^{3} \tilde{a}_{i}=37-11 \alpha$

$\sum_{j=1}^{3} \tilde{b}_{j}=21+9 \alpha$

? $=0.8$ is found from $37-11 \alpha=21+9 \alpha$. The satisfactory level $(\alpha)$ will be in $[0, \alpha]=[0,0.8]$ interval. Therefore, quantity in the artificial demand center is

$b_{j}^{4}=\sum_{i} \tilde{a}_{i}-\sum_{j} \tilde{b}_{j}=16-20 \alpha$

so in this problem, the total available quantity in the sources is exactly equal to the total quantity required by the destinations, that is, the problem is balanced.

Table.2: Fuzzy Transportation Problem Values

\begin{tabular}{|c|c|c|c|c|}
\hline Supply Demand & $b_{j}^{1}=2+3 \alpha$ & $b_{j}^{2}=14+\alpha$ & $b_{j}^{3}=5+5 \alpha$ & $b_{j}^{4}=16-20 \alpha$ \\
\hline$a_{i}^{1}=11-2 \alpha$ & $\tilde{\mathrm{C}}_{11}=5-2 \gamma$ & $\tilde{\mathrm{C}}_{12}=7-\gamma$ & $\tilde{\mathrm{C}}_{13}=11-5 \gamma$ & $\tilde{\mathrm{C}}_{14}=0$ \\
\hline$a_{i}^{2}=12-4 \alpha$ & $\tilde{\mathrm{C}}_{21}=9-2 \gamma$ & $\tilde{\mathrm{C}}_{22}=15-6 \gamma$ & $\tilde{\mathrm{C}}_{23}=18-8 \gamma$ & $\tilde{\mathrm{C}}_{24}=0$ \\
\hline$a_{i}^{3}=14-5 \alpha$ & $\tilde{\mathrm{C}}_{31}=13-4 \gamma$ & $\tilde{\mathrm{C}}_{32}=16-7 \gamma$ & $\tilde{\mathrm{C}}_{33}=10-\gamma$ & $\tilde{\mathrm{C}}_{34}=0$ \\
\hline
\end{tabular}

The breaking points of ?'s are intersection points of lines $\tilde{C} i j=\tilde{C} i j\left(\tilde{X}_{i j}\right)$. Breaking values of ? in $[0,1]$ interval are $0,1 / 4,2 / 3$ and 1 . Intervals among these iterative values are $[0,1 / 4],[1 / 4,2 / 3]$, $[2 / 3,1]$.Now for interval 0 [ $[0,1 / 4]$, let us solve the transportation problem with fuzzy quantity. 
Let ? = 0 as representative of the first interval for ?. Then, the obtained optimal solution is given in Table 3.

Table.3: Optimal Solutions for $(\alpha)$ Level

\begin{tabular}{|c|c|c|c|c|}
\hline Supply Demand & $b_{j}^{1}=2+3 \alpha$ & $b_{j}^{2}=14+\alpha$ & $b_{j}^{3}=5+5 \alpha$ & $b_{j}^{4}=16-20 \alpha$ \\
\hline$a_{i}^{1}=11-2 \alpha$ & & $11-2 \alpha$ & & $\tilde{\mathrm{C}}_{14}=0$ \\
\hline & $\tilde{\mathrm{C}}_{11}=5-2 \gamma$ & $\tilde{\mathrm{C}}_{12}=7-\gamma$ & $\tilde{\mathrm{C}}_{13}=11-5 \gamma$ & $7-10 \alpha+\mathrm{t}$ \\
\hline$a_{i}^{2}=12-4 \alpha$ & $2+3 \alpha$ & $3+3 \alpha-\mathrm{t}$ & & $\tilde{\mathrm{C}}_{24}=0$ \\
\hline & $\tilde{\mathrm{C}}_{21}=9-2 \gamma$ & $\tilde{\mathrm{C}}_{22}=15-6 \gamma$ & $\tilde{\mathrm{C}}_{23}=18-8 \gamma$ & $9-10 \alpha-t$ \\
\hline$a_{i}^{3}=14-5 \alpha$ & & $+\mathrm{t}$ & $5+5 \alpha$ & $\tilde{\mathrm{C}}_{33}=10-\gamma$ \\
\hline
\end{tabular}

For the values in intervals, the values of ? in the supply and demand centers are distributed homogenous to cell that are occurred optimal transportation problem. The first value ? that violates non-negative condition of optimal solution is $\tilde{X}_{24}=7-10$, ? $=0$. Thus, the present optimal solution is valid for interval ?. 2? [0,7/10].

$0 \leq \gamma \leq \frac{1}{4}$ and $\frac{7}{10} \leq \alpha \leq \frac{8}{10}$

In Table 4, the obtained solutions for interval ?. 2 [7/10, 8/10] are optimal and feasible. The obtained optimal solutions for intervals of $\left[\begin{array}{l}\text { [ } \\ {[1 / 4,2 / 3]}\end{array}\right.$

Table.4: The Optimal and Feasible Solutions for Interval $\alpha[7 / 10,8 / 10]$

\begin{tabular}{|c|c|c|c|c|}
\hline Supply Demand & $b_{j}^{1}=2+3 \alpha$ & $b_{j}^{2}=14+\alpha$ & $b_{j}^{3}=5+5 \alpha$ & $b_{j}^{4}=16-20 \alpha$ \\
\hline$a_{i}^{1}=11-2 \alpha$ & & $11-2 \alpha$ & & \\
\hline$a_{i}^{2}=12-4 \alpha$ & $\tilde{\mathrm{C}}_{11}=5-2 \gamma$ & $\tilde{\mathrm{C}}_{12}=7-\gamma$ & $\tilde{\mathrm{C}}_{13}=11-5 \gamma$ & $\tilde{\mathrm{C}}_{14}=0$ \\
\hline & $2+3 \alpha$ & $10-7 \alpha$ & & $\tilde{\mathrm{C}}_{24}=0$ \\
\hline$a_{i}^{3}=14-5 \alpha$ & $\tilde{\mathrm{C}}_{21}=9-2 \gamma$ & $\tilde{\mathrm{C}}_{22}=15-6 \gamma$ & $\tilde{\mathrm{C}}_{23}=18-8 \gamma$ & $5+5 \alpha$ \\
\hline & & $-7+10 \alpha$ & $\tilde{\mathrm{C}}_{33}=10-\gamma$ & $\tilde{\mathrm{C}}_{34}=0$ \\
\hline
\end{tabular}

Table.5: The Optimal and Feasible Solutions for Interval $\gamma[1 / 4,2 / 3] \& \quad[2 / 3,1]$

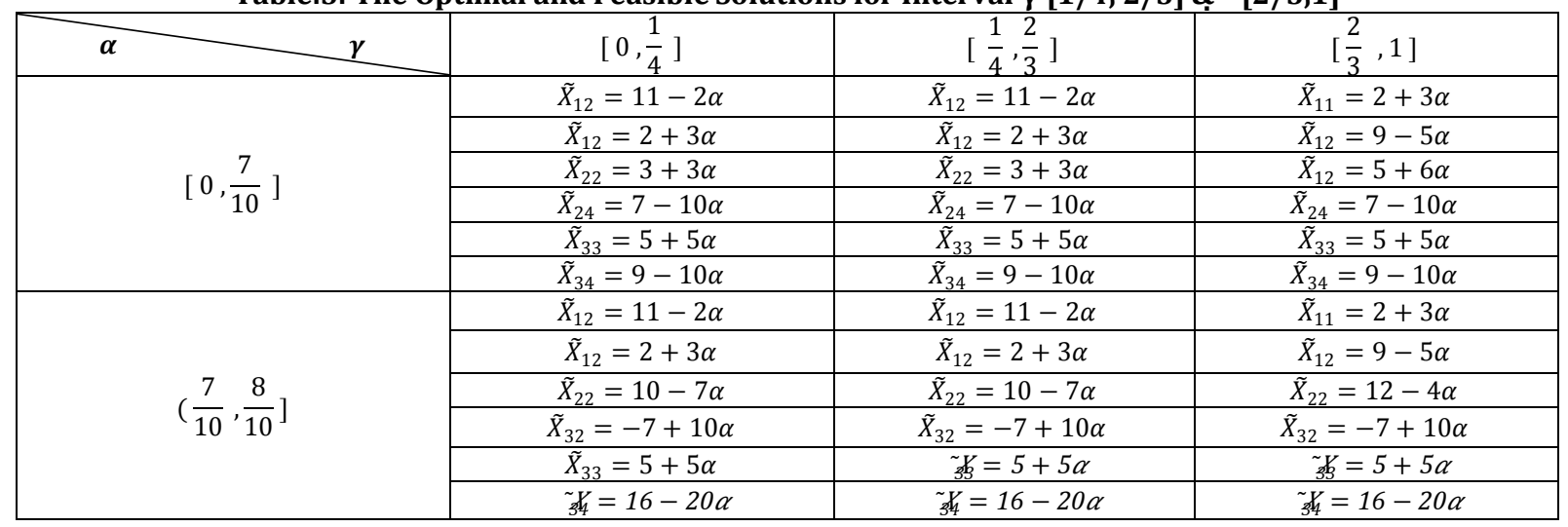

\section{Conclusion}

In this paper the author has shown that there is an optimal solution that each interval constituting by the iterative breaking points. Like stochastic order, after the breaking points of the satisfactory level that are changed, the values of transportation costs are respectively determined. However, It is transferred a new optimal solution by using duality while the optimal solutions lose 
Hossein Abdollahnejad Barough / TJMCS Vol .2 No.1 (2011) 184-194

their feasibility because of the satisfactory level that depends on quantities' increases. The breaking points of satisfactory levels depend on quantities and the changed optimal solutions are obtained successively. Because of the special structure of the problem, all of these operations are realized and the optimal solutions are obtained for all fuzzy cases that may be encountered in future to the transportation problem.

\section{References}

[1] Ahlatcioglu, M., Sivri, M., Güzel, N., "Transportation of the fuzzy amounts using the fuzzy cost", Journal of Marmara for Pure and Applied Sciences, Istanbul, Turkey, Vol.18, 2002.

[2] Ammar, E.E., Youness. E., "A Study on multi-objective transportation problem with fuzzy numbers", Applied Mathematics and Computation, Vol.16, No.2, pp. 241-253, 2005.

[3] Chanas, S., Kulej, M., "A fuzzy linear programming problem with equality constrains", Control and Cybernetics, Vol.13, pp. 195-201, 1984.

[4] Chanas, S., Kolodziejczyk, W., Machaj, A., "A fuzzy Approach to the Transportation Problem", Fuzzy Sets and System, Vol.13, pp. 211-221, 1984.

[5] Chanas, S., Kuchta, D., "Fuzzy integer transportation problem", Fuzzy Sets and Systems, Vol. 98, pp. 291298, 1998.

[6] Geetha, S., Nair, K.P., "A Stochastic Bottleneck Transportation Problem", Journal of Operations Research, Vol.45, No.5, pp. 583-588, 1994.

[7] Gen, M., Ida, K., Li, Y., "Solving Bicriteria Solid Transportation Problem", Humans, information and Technology. IEEE International Conference, Vol.2, pp.1200-1207, 1994.

[8] Greig, D.M., “Optimization”, Lonman Group Limited, London, pp. 100-111, 1980.

[9] Kikuchi, S., "A method to defuzzify the fuzzy number: Transportation problem application", Fuzzy Sets and Systems, Vol.116, pp. 3-9, 2000.

[10] Kwak, N.K., "Mathematical Programming with Business Applications", McGraw-Hill Book Company, New York, pp: 88-149, 1973.

[11] Lai, Y.J., Hwang, C.L., "Fuzzy Mathematical Programming”, Springer-Verlag, Berlin, 1992.

[12] Li, Y., Ida, K., Gen, M., Kobuchi, R., "Neural Network Approach for Multi-criteria Solid Transportation Problem", Computers \& Industrial Engineering, Vol.33, No. (3-4), pp.465-468, 1997.

[13] Liu, S.-T., Kao, C., "Solving Fuzzy Transportation Problems based on extension principle", European Journal of Operational Research, Vol.153, pp.661-674, 2004.

[14] OhEigeartaigh, M., "A fuzzy transportation algorithm", Fuzzy Sets and Systems, Vol.8, pp.235-243, 1982.

[15] Ringuest, J.L., Rink, D.B., "Interactive solutions for the linear multi-objective transportation problem", European Journal of Operations Research, North-Holland, Vol.32, pp. 96-106, 1987.

[16] Wahed, W.F.A.E., "A multi-objective transportation problem under fuzziness", Fuzzy Sets and Systems, Vol.117, pp. 27-33, 2001.

[17] Wahed, W.F.A.E., Lee, S.L., "Interactive fuzzy goal programming for multi objective transportation problems”, Omega, Vol.34, pp.158-166, 2006. 